\title{
The Research of Computer Database Technology Application in Information Management
}

\author{
XiaoJun JIANG ${ }^{1,}$, , Yuye ZHAO $^{2}$ \\ ${ }^{1}$ College of Extended Education, Jilin Teachers Institute of Engineering and Technology, \\ Changchun Jilin 130052, China \\ ${ }^{2}$ Langfang Teachers University, hebei langfang 065000,China \\ a jiangxiaojun@yeah.net
}

Keywords: Database; Information management; SQL

\begin{abstract}
As the core technology and an important foundation of computer database system, the construction of data model for the development of a variety of data management software provides effective reference for the design. Structure mode in the construction of the database structure, different data model to divide the database structure to hierarchical databases, relational database and network database types, which greatly improved the adaptability of the database structure and the computer system, and also makes the computer database and the application scope of applications have been further widened. In this paper, the computer database technology in the application of information management has carried on the detailed research, this paper analyzed the characteristics of database system and its application in information management present situation and put forward the improving its application in the information management advice.
\end{abstract}

\section{Introduction}

With the development of the domestic computer technology and network communication technology, database technology has become an information society on a large amount of data organization and management of the important technology and software technology, is the foundation of the network information management system. Database technology is an important part of modern information science and technology, is the core of computer data processing and information management system. At the same time, the database and information management system are complementary to each other, are inseparable whole, the combination of the two is the basis of the information construction and the safeguard, also is the important power to promote the development of science and technology [1]. Database technology to research and solve in the process of the computer information processing of large amounts of data organization and storage problems effectively, reduce data storage redundancy in the database system, realize data sharing, data security and efficiently retrieve data and process the data. The combination of computer technology and database, create a very broad market prospects for development and application range are also constantly expanding, involving the domestic industry, agriculture, business and so on all walks of life, the application of the computer database technology provides the domestic many industry information management technology, promote industrial productivity and efficiency [2].

\section{The analysis of application of computer database in information management system}

In computer data are stored in the database is organized, not chaos unrelated, and data in the database information in computer are greatly related each other, is with the corresponding structure connection between individual data, in a certain structure form has its own relations, in the same set of data characteristics are the same. Database to make the enterprise internal data sharing, data for the enterprise internal management has brought a lot of convenient, so can share the database is the most important factor. Computer database system will be according to the meaning of the user, will be automated delete useless information, and for the convenience of user query of data information, 
improve the efficiency of data query. Database management system can manage the user's data, the user can according to the data you want to enter a query, at the same time, and the user can also according to the database design database features they want [3-4].

The application of computer database information management gradually extensive. At present, the database information management and they are widely used in each big industry and the development of information technology market has great potential. The application of the computer database technology, has provided the safeguard for many enterprise data management, enterprise internal information data for the effective management, enhance the ability of enterprise's data processing, improve the management level of the enterprise, thus to promote the development of enterprise. So nowadays many companies are identified with the database information management system, thus laid a foundation for the development of the computer database technology.

The safety performance of the computer database technology gradually strengthened. The application of computer database system for the security of enterprise information management provides a great convenience, effectively guarantee the enterprise information data security, a database information system can automatically backup data of the information management, and prevent data accidentally deleted. With the continuous development of computer information technology, the database information technology unceasing development, its safety performance after continuously perfect, to the enterprise internal information security management is higher. Can effectively prevent data loss, improve the management efficiency of enterprises.

Computer database technology in the application of computer management gradually extensive. Database information technology system in the application of life has been widespread, each big enterprise's attention. And the design of the database information system more humanized, to enhance the flexibility, operation is simple, the user can easily to finish using the database information. With the continuous development of multimedia information industry, the requirement of database information processing ability have been enhanced, so for the application of database technology will be wider attention.

\section{Key technology of computer database}

As an important part of the modern information science and technology, database technology is the core of computer data processing and information management system. How to study and solve it effectively organize large amounts of data, computer information processing in data storage, reduce the data redundancy, realize the sharing of the data in the database system, ensure the safety of data and fast retrieval and data processing [5]. The data is the object database technology research and management, which will limit the specific contents of the database technology involved should include: the specified structure to establish the corresponding database and data warehouse can be realized by unified data organization and management; in the design of database management system and data mining system, should be to achieve a variety of functions the user to add, modify, delete data in a database, processing, analysis, understanding, report and print; the use of application management system finally realizes the processing, analysis and understanding of the data.

SQL Server is a large and complex software packages. It is the core engine of a strong relationship between the data, but also contains related services, tools and the development of technology, analysis of main components as shown in figure 1.

The database engine is the core of database management system, SQL Server database engine itself consists of two main components: the query processor (the query processor) and the storage engine (storage engine). The query processor uses the SQL statement to express its SQL statement can be divided into atomic other measures can serial or parallel query processing. In addition, it will select a query plan in a certain range, and the proper use of index. The storage engine task is responsible for the operation of the database physical structure, organization structure of the database files, which directly access the data on the disk to complete the corresponding function. 


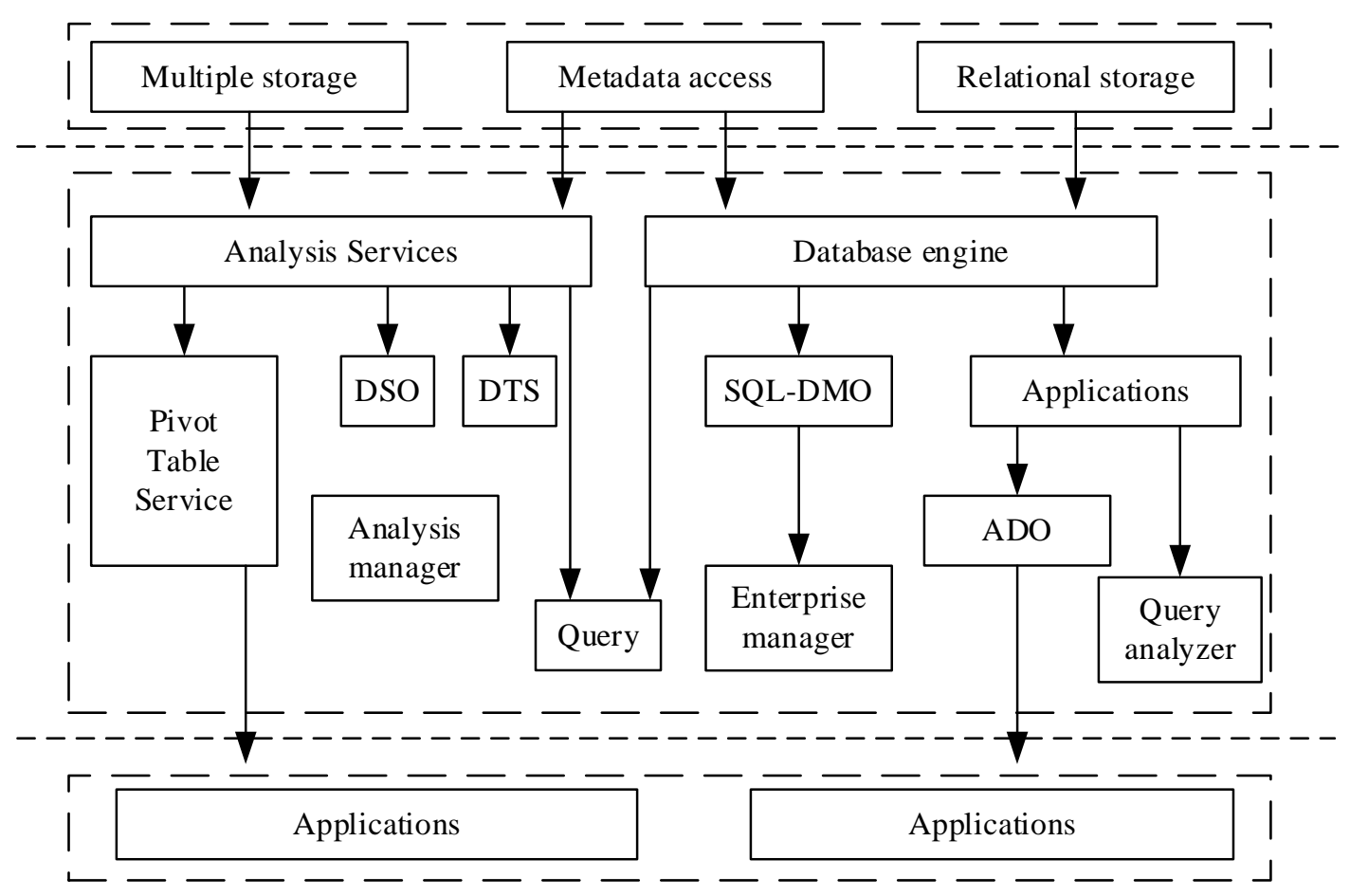

Figure 1.Service component of computer database

\section{Research of SQL database model}

The core and foundation of database system is a data type, but also in the database abstraction of the real world. General data model consists of three elements, namely, data structure, data operation and data integrity constraints. The main data structure is used to describe the relationship between the static characteristics of the data, the data structure and data, data operation refers to the existing data in the database query, modify, delete or add a variety of new data access methods, and may also include data access rules; data integrity constraints, a set of integrity rules. SQL (Structured Query Language) is a kind of relation algebra and calculus of relations between the structured query languages (SQL), its function is not only a query. SQL database is a universal language, very powerful. The SQL statement can access data from a relational database, you can also use the SQL statement to create the database, modify data, and to increase the data, etc., SQL language is widely used, is the main characteristics, easy to use and easy to learn, is an industry standard language for database operation. For example, DB2, ORACLE relational database products have been realized in SQL language. Support the relational database SQL language level three model structure, as shown in figure 2. The contact model of view, a part of the base table, the basic table model, connection files stored within the model.

Basic user table, view query or related operations can be written by SQL language, the basic table and view is consistent, the SQL said. The basic form of SQL is corresponding to a relationship, is itself an independent existence. One or more basic table corresponds to a storage file, a table can have multiple index and storage file also contains the index. Relational database model is composed of a storage file logical structure and physical structure of the files stored on the user is transparent, and is arbitrary. The view itself is not stored in the database, it comes from one or several basic table. Only in database view has a characteristic is, the corresponding definition, but not stored view corresponding data, database derived basic deposit corresponding Datasheet view, can be considered to be essentially a view is a virtual table. From the concept and the basic table, view is consistent, is for the user to define a view in the view on the basis. 


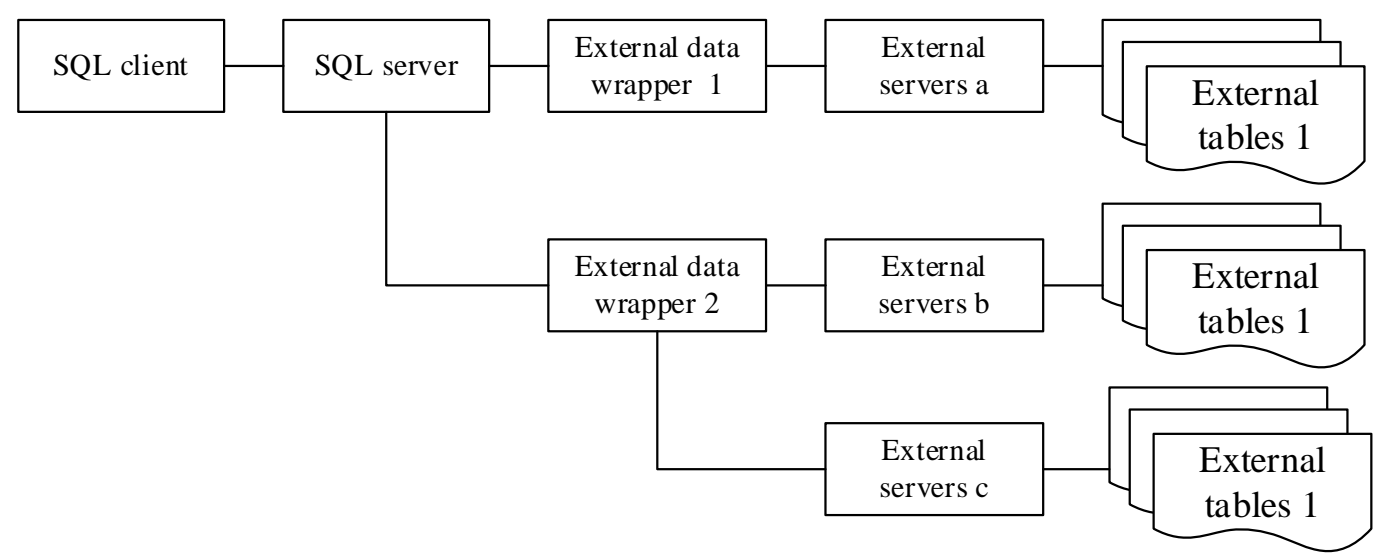

Figure 2. SQL relational database model structure

\section{Conclusion}

Computer database system in the management of the enterprise operational data for corresponding effective at the same time, also for the enterprise operating information provides a powerful security, this mainly displays in the database backup and recovery process of enterprise information system and data replication and copy under zero investment cost, etc., the computer database system in the application of information management not only reduced the computer system information mistaken delete influence on information management, also greatly improve the safety and practicability of the modern information management. With the deepening of the database and computer information management application, we also found some problems to be solved, such as database security protection needs to be improved, the updating of the database theory needs further in practice to prove, our ongoing research is needed to promote better and faster development of database technology, promote the information management better, more secure applications.

\section{Reference}

[1] T.J. Chen: Journal of Management Studies, Vol.40 (2009) No.5, p. 1107.

[2] N. Bozionelos: International Journal of Organizational Analysis, Vol.11 (2010) No.1, p. 41.

[3] S. Granger: Tesol Quarterly, Vol.37 (2012) No.3, p. 538.

[4] S.F. Peregoy, O.F. Boyle, and S. Phillabaum: TESOL Quarterly, Vol.41 (2011) No.1, p. 214.

[5] Cai. Jigang: Foreign Language Teaching and Research, Vol.1 (2008), p. 005. 